\section{PerFRACTION ${ }^{T M}$}

3D Pre-Treatment QA and In-Vivo Monitoring

- Fraction $\boldsymbol{O}^{\mathrm{TM}}$ Pre-treatment phantom-free QA with the independence and peace of mind of EPID measurements

- Fraction $n^{\text {TM }}$ In-vivo QA for every treatment catches patient and machine errors

- See which clinical goals pass and fail for each daily fraction

- Automated DICOM file capture, analysis, and email notifications for failure

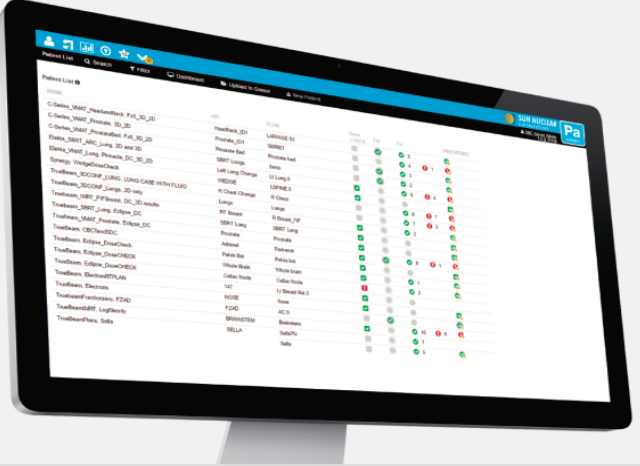

\section{Stereotactic QA}

New Solutions

\section{SRS MapCHECK}

Small Field Digital Film Replacement for Patient Specific QA \& End-to-End Testing

- Designed to insert into StereoPHAN ${ }^{m m}$ and run on SNC Patient ${ }^{\text {tw }}$ software

Detector Spacing: $2.47 \mathrm{~mm}$; Detector Resolution: $0.47 \mathrm{~mm}$ diameter

- Measures field sizes as small as $5 \mathrm{~mm}$; 5 diodes in $5 \mathrm{~mm}$ cone

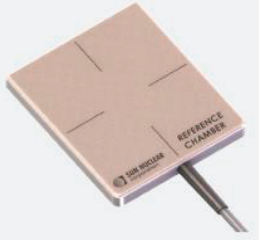

Reference Detector

An Out-of-Field Reference Detector for Water Tank Scanning

Measures LINAC head leakage, enabled by large-volume ion chamber

Used during photon beam commissioning measurements of any field size

Eliminates scanning chamber interference in small SRS fields

- Volume: 39cc; 2-meter cable with triaxial connector
DoseCHECK ${ }^{\text {Tm }}$

Independent Secondary 3D Dose Calculations

- Automated, TPS grade dose volume generation

- Efficient dose-to-dose evaluation

- Seamless integration with the PerFRACTION ${ }^{\mathrm{TM}}$ solution for patient QA

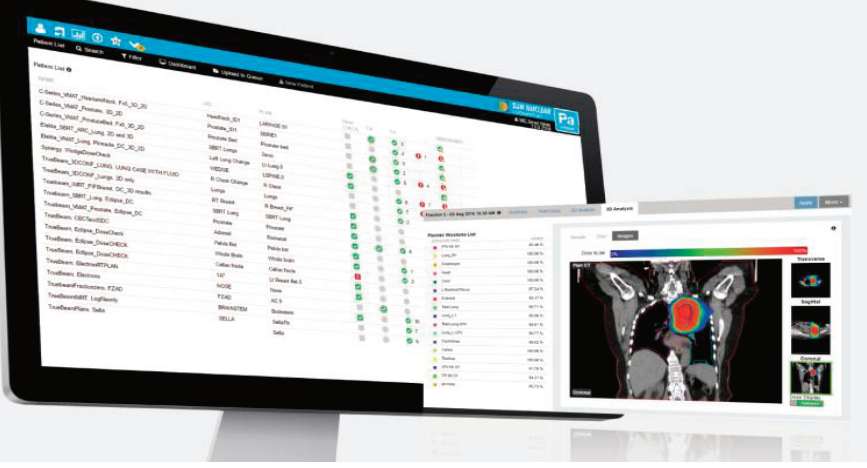

SNC Machine ${ }^{\mathrm{TM}}$

TG-142/VMAT Imaging and Mechanical QA

- Automated workflow captures and analyzes files - no further work required

- Supports most common mechanical and imaging phantoms

- Trend any test parameter against any other test parameter for any number of machines

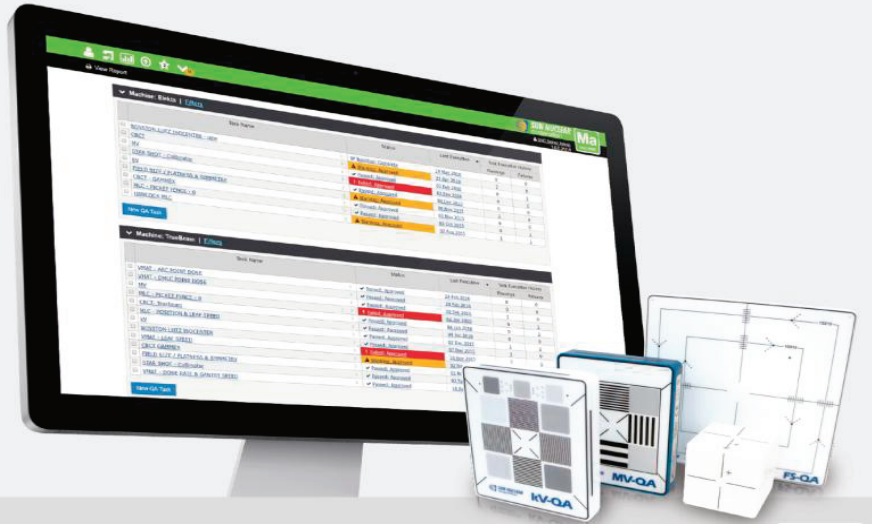




\title{
Review of National Diagnostic Reference Levels for Interventional Procedures
}

\author{
Min Young Lee ${ }^{(\mathbb{D})}$, Jae Kwon ${ }^{(\mathbb{1})}$, Gang Woo Ryu ${ }^{\mathbb{D}}$, Ki Hoon Kim ${ }^{(\mathbb{D}}$, Hyung Woo Nam ${ }^{\mathbb{1}}$, Kwang Pyo Kim ${ }^{(\mathbb{D})}$ \\ Department of Nuclear Engineering, Kyung Hee University, Yongin, Korea
}

Received 6 December 2019 Revised 19 December 2019

Accepted 23 December 2019

Corresponding author

Kwang Pyo Kim

(kpkim@khu.ac.kr)

Tel: 82-31-201-2560

Fax: 82-31-202-8106
Diagnostic reference level (DRL) is employed to optimize the radiation doses of patients. The objective of this study is to review the DRLs for interventional procedures in Korea and abroad. Literature review was performed to investigate radiation dose index and measurement methodology commonly used in DRL determination. Dose area product (DAP) and fluoroscopy time within each major procedure category were systematically abstracted and analyzed. A wide variation was found in the radiation dose. The DAP values and fluoroscopy times ranged $0.01-3,081 \mathrm{~Gy} \cdot \mathrm{cm}^{2}$ and $2-16,878$ seconds for all the interventional procedures, 8.5-1,679 $\mathrm{Gy} \cdot \mathrm{cm}^{2}$ and $32-5,775$ seconds for the transcatheter arterial chemoembolization (TACE), and $0.1-686 \mathrm{~Gy} \cdot \mathrm{cm}^{2}$ and $16-6,636$ seconds for the transfemoral cerebral angiography (TFCA), respectively. The DRL values of the DAP and fluoroscopy time were $238 \mathrm{~Gy} \cdot \mathrm{cm}^{2}$ and 1,224 seconds for the TACE and $189 \mathrm{~Gy} \cdot \mathrm{cm}^{2}$ and 686 seconds for the TFCA, respectively. Generally, the DRLs of Korea were lower than those of other developed countries, except for the percutaneous transluminal angioplasty with stent in arteries of the lower extremity (LE PTA and stent), aneurysm coil embolization, and Hickman insertion procedures. The wide variation in the radiation doses of the different procedures suggests that more attention must be paid to reduce unnecessary radiation exposure from medical imaging. Furthermore, periodic nationwide survey of medical radiation exposures is necessary to optimize the patient dose for radiation protection, which will ultimately contribute to patient dose reduction and radiological safety.

Keywords: Interventional radiology, Radiation protection, Diagnostic reference level, Patient dose, Radiation exposure

\section{Introduction}

Interventional radiology is a medical specialization that involves performing a range of imaging procedures to obtain images of the inside of the body of a patient. Interventional radiologists conduct various procedures, such as treating tumors, taking organ biopsies, or placing stents, by inserting miniature instruments and thin catheters into the body via an artery or a vein. Interventional radiology has the advantage of reducing medical expenses as well as the pain from invasive procedures (e.g., surgery). Currently, the use of interventional radiology is rapidly increasing with the increase in average life expectancy and technological development. ${ }^{1)}$

Fig. 1 shows the number of interventional radiology procedures performed during the period of 2007-2011, as reported in a 2013 domestic study. The use of interventional radiology increased by approximately $12 \%$ per year, from 22,000 in 2007 to approximately 35,000 in 2011. These results included only the procedures involving imaging tests performed during interventional radiology in that period. Therefore, the actual number of procedures is expected to 


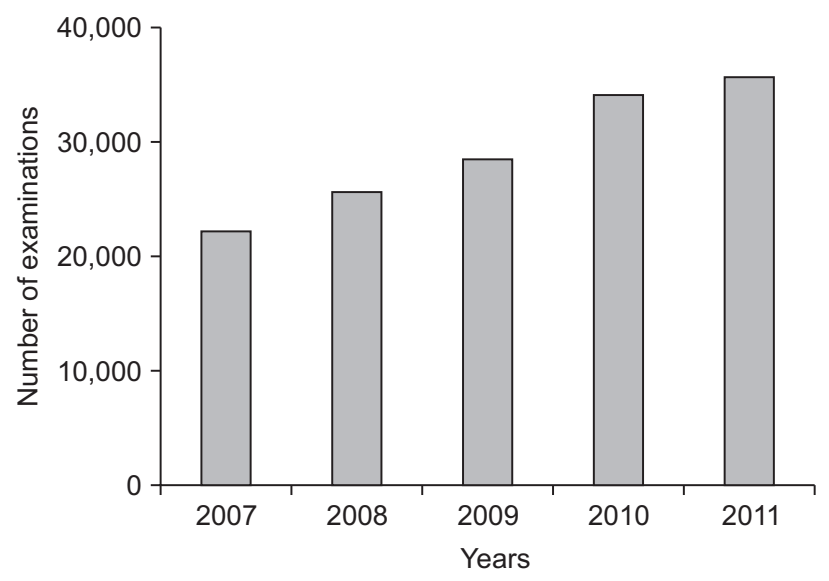

Fig. 1. Number of interventional procedures per year in Korea.

be larger than the above value. ${ }^{2)}$ In addition, interventional radiology requires a longer time and relatively higher radiation doses than other diagnostic radiology examinations. Considering its increased usage and high radiation dose, currently, radiation protection and patient dose management are important in interventional radiology. Further, because interventional radiology is an important procedure directly related to the survival of a patient, it is inappropriate to apply extremely strict measures on the radiation exposure received by the patient. However, efforts to optimize the procedure and measures for patient radiation protection are needed.

Guidance or recommendations for radiation protection in diagnostic radiology are provided by the International Commission on Radiological Protection (ICRP). The ICRP uses the following approach for radiation protection of patients. Generally, radiation exposure of a patient during radiology, such as interventional radiology, is higher than that in daily life; however, the patient also receives the medical benefits from the procedure. The ICRP states that radiation in these areas of medical care must be used for the benefit of the patient and the dose should be optimized. ${ }^{3)}$ Summarizing, the basic principle of patient protection in medical exposure is to maximize the medical benefits of radiation use while keeping the dose as low as reasonably achievable considering economic and social factors. ${ }^{4-6)}$

The measured or irradiated radiation dose at a radiological examination facility differs according to the radiologist, patient, and hospital, even if the same procedure is used.
Therefore, the necessity of setting a reference radiation dose for a patient has emerged. As interventional radiology is a procedure that is directly related to the life of the patient, it is inappropriate to apply extremely strict controls. Therefore, the equipment or institutions that administer extremely high or extremely low radiation doses need to be identified, and the doses should be optimized.

A reference level was introduced to implement radiation dose reduction and establish a reference standard. This concept was first introduced in ICRP Publication $60 .^{3)}$ Subsequently, the ICRP recommended the use of diagnostic reference levels (DRLs) for medical purposes in ICRP Publication 73, 8 and ICRP Supporting Guidance 2.,7)

The International Atomic Energy Agency (IAEA) also endorses establishing DRLs, the recommended radiation dose levels for patients in accordance with the medical conditions, in each country. ${ }^{8)}$ According to these guidelines, various countries, such as the United Kingdom, the United States, and European countries, are conducting radiation exposure status surveys and DRL studies on various diagnostic procedures, to optimize patient dose management.

In Korea, the Ministry of Food and Drug Safety (MFDS) and the Center for Disease Control and Prevention have conducted DRL determination studies on various diagnostic procedures by measurements or surveys. ${ }^{9,10)}$ The scope of the investigation for DRL determination ranges from hospital units to regional and national levels. Considering the increased usage of interventional radiology and high radiation doses, it is necessary to investigate and analyze studies on DRL determination for domestic and international interventional radiology in terms of patient radiation protection and patient dose management.

The purpose of this study was to review the DRLs for interventional radiology in Korea and abroad. To this end, we first examined the methodology of DRL determination. The radiation dose index and measurement or irradiation methodology commonly used in DRL determination for interventional radiology were investigated. In addition, studies on the DRL determination for domestic and international interventional procedures were examined, and the results were compared and analyzed. 


\section{Diagnostic Reference Level and Radiation Dose for a Diagnostic Reference Level}

\section{Diagnostic reference level}

In this study, we investigated the DRL determination methodology and the radiation dose indicator used to determine a DRL. A DRL may be used as an indicator for evaluating how much radiation dose is used for a patient. DRL determination can be conducted by large-scale radiation dose surveys or measurements across hospitals, regions, or a country. A DRL is generally determined as the 75th percentile level of the measured or surveyed radiation dose distribution. It can be applied in various diagnostic procedures, such as computed tomography (CT), nuclear medicine, and interventional radiology. Fig. 2 presents an example of the radiation dose distribution and DRL. From Fig. 2, it can be seen that although most radiation doses are low, some are extremely high. The identification and corrective actions of facilities or equipment that administer abnormally high radiation doses can significantly reduce the radiation dose of a patient. DRL determination should not end as a one-time event; the DRL needs to be measured and examined periodically and updated. In the UK, where early studies of DRL determination have been conducted, it has been found that the radiation dose decreases over time. ${ }^{11)}$

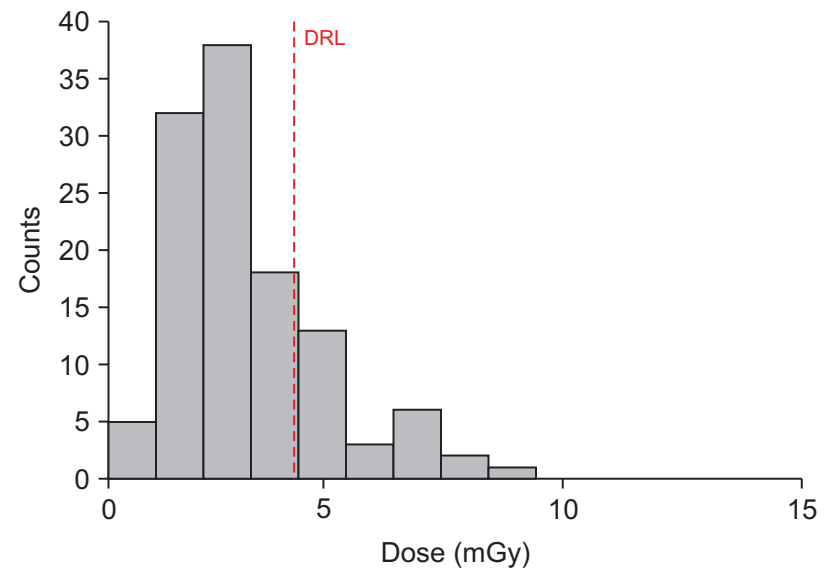

Fig. 2. Dose distributions of diagnostic radiology and diagnostic reference level (DRL).

\section{Radiation dose for interventional procedure diagnostic reference level}

The measurement or survey methodology for the radiation dose indicator was investigated. The ICRP, International Commission on Radiation Units and Measurements (ICRU), and IAEA have proposed radiation dose units for DRLs. ${ }^{6,12,13)}$ The ICRP proposes as conditions of the patient radiation dose indicator that the assessment of the amount of ionizing radiation used in a procedure and easy measurement or determination of the indicator. ${ }^{6)}$ The ICRU and IAEA recommend using a physical quantity that can be represented by air kerma as the unit of the radiation dose of a DRL. ${ }^{12,13)}$ In general, the dose area product (DAP) and reference air kerma (RAK) are used for the measurement of the radiation dose of a patient in an interventional procedure, because they are easy to be calculated and measured. Further, the fluoroscopy time is employed as an additional indicator.

The concept of DAP is displayed in Fig. 3. The DAP is defined as the product of the absorbed dose that does not include the backscattering effects in air measured at the same distance from the focal point and the area of the X-ray irradiation field. The DAP can provide information about the irradiation area in which a patient is actually exposed. The intensity of the radiation decreases in inverse proportion to the square of the distance on going further from the focal point. In contrast, the irradiation area of the radiation increases in proportion to the square of the distance; thus, the above two effects cancel each other. Therefore, the DAP

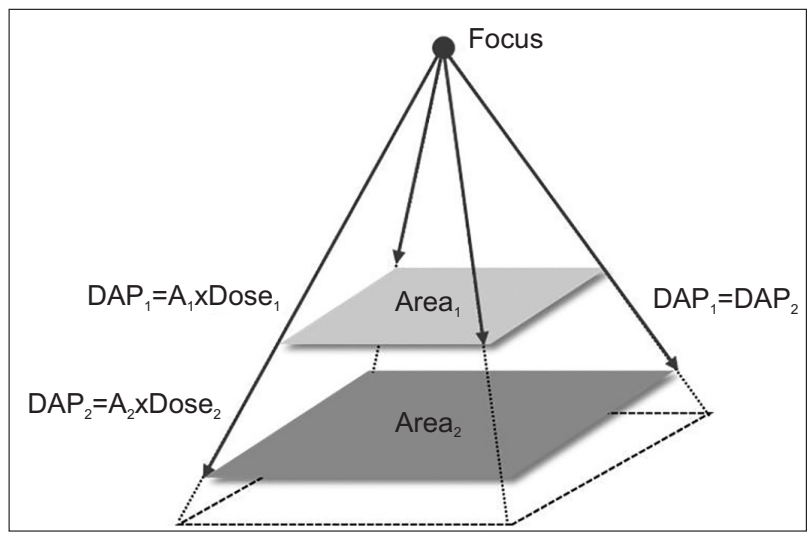

Fig. 3. Concept of the dose area product (DAP) measurement. 
can be defined at any position on the beam axis between the X-ray focal point and the patient. It can be derived from direct measurements or calculations. Direct measurement of the DAP employs an area dosimeter at the outlet of the X-ray tube. Recently used interventional procedure equipment utilize the area dosimeter at their outlets or use a calculation formula to display the DAP value employed for the patient during the procedure on a screen.

The conceptual diagram of the RAK measurement is shown in Fig. 4. The RAK is a value indicating the radiation dose incident on the skin surface of a patient during an interventional procedure or fluoroscopy. It is measured at a point in space located at a certain distance from the focal point. In general, in the case of a $\mathrm{C}$-arm, it is measured at a patient incident reference point $15 \mathrm{~cm}$ away from the center of rotation on the X-ray beam axis in the focal point direction. ${ }^{14)}$ This incident reference point is similar to the point where the skin of the patient is located, assuming a standard sized patient is lying on the bed. Therefore, the RAK can be used to indirectly determine the skin dose received by a patient. Various terms are used in different organizations to indicate the RAK, such as reference air kerma, cumulative air kerma, reference point air kerma, and cumulative dose.

The fluoroscopy time is the total time the fluoroscopy was performed during the interventional procedure. Because the radiation dose is directly proportional to the time of use, the fluoroscopy time is a significant contributor to the exposure dose. In addition, it has the advantage of

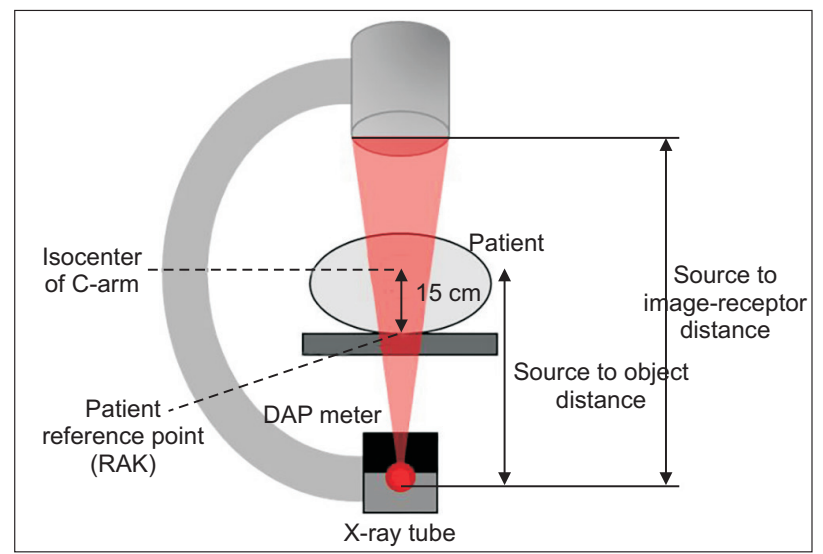

Fig. 4. Concept of the reference air kerma (RAK) measurement. DAP, dose area product. easily collecting information because it does not require a separate measuring equipment. However, the fluoroscopy time can be used only as a reference because it does not reflect the intensity of the injected X-ray, performance of the device, or irradiation area. Other studies suggest that the fluoroscopy time alone is not a useful indicator of the dose of a patient during a procedure and that we should not examine it separately for patient dose investigation. ${ }^{15-19)}$

\section{Nationwide Diagnostic Reference Level for Interventional Procedures}

1. UK

In the UK, DRL determination studies on diagnostic radiology are actively conducted by the Health Protection Agency (HPA). The UK Department of Health and Social Security periodically collects exposure dose information regarding diagnostic radiology and determines the DRL based on the collected data. A DRL determination and exposure studies for interventional procedures in the UK were conducted in 2005 and 2010. ${ }^{11,20)}$ In a 2012 study, data on various diagnostic procedures, including interventional procedures and diagnostic radiation, were collected from January 2006 to December 2010 at a total of 320 medical institutions nationwide for patient dose investigation. This number represents approximately $25 \%$ of the hospitals with diagnostic radiography equipment in the UK. In the UK, data on six major procedures, such as biliary intervention and facet joint injection, were collected. Data were collected not only on the DAP and the fluoroscopy time, but also on patient information, such as age and weight. The information collection for the DAP was based on approximately 15,000 and 14,000 cases of patients, respectively. Each exposure dose information was collected by filling out questionnaires, and the data verification was performed by a statistical analysis program. Table 1 summarizes the DRL and survey results for the DAP and the fluoroscopy time during the interventional procedures in the UK as of $2010 .^{11)}$ 


\section{USA}

In the US, medical radiation exposure status surveys were conducted driven by the Food and Drug Administration (FDA) and the Society of Interventional Radiology
(SIR). ${ }^{21)}$ From 2003 to 2009, these organizations performed a study of the patient dose measurement and evaluation of 21 interventional procedures called "Radiation Doses in Interventional Radiology Procedures (RAD-IR) Study". To this end, the radiation dose data were collected from a total

Table 1. Dose area products (DAPs) and fluoroscopy times for interventional procedures in the UK (2012)

\begin{tabular}{|c|c|c|c|c|c|c|c|c|}
\hline \multirow{2}{*}{$\begin{array}{l}\text { Radiation } \\
\text { dose unit }\end{array}$} & \multirow{2}{*}{ Procedure } & \multicolumn{3}{|c|}{ Measurement (n) } & \multirow{2}{*}{ Mean } & \multirow{2}{*}{ Minimum } & \multirow{2}{*}{ Maximum } & \multirow{2}{*}{ DRL } \\
\hline & & Hospital & Room & Patient & & & & \\
\hline \multirow[t]{7}{*}{$\mathrm{DAP}\left(\mathrm{Gy} \cdot \mathrm{cm}^{2}\right)$} & Biliary intervention & 10 & 22 & 279 & 33 & 6 & 111 & 43 \\
\hline & Facet joint injection & 20 & 30 & 2,720 & 4 & 0.2 & 16 & 6 \\
\hline & Hickman line insertion & 21 & 37 & 829 & 2 & 0.1 & 10 & 3 \\
\hline & Nephrostomy & 24 & 31 & 464 & 8 & 0.5 & 24 & 13 \\
\hline & Oesophageal stent & 15 & 24 & 199 & 13 & 3 & 76 & 13 \\
\hline & Pacemaker (permanent) & 31 & 78 & 5,062 & 7 & 0.2 & 34 & 7 \\
\hline & PTCA 1 stent & 14 & 39 & 5,805 & 34 & 12 & 81 & 40 \\
\hline \multirow{7}{*}{$\begin{array}{l}\text { Fluoroscopy } \\
\text { time (s) }\end{array}$} & Biliary intervention & 9 & 21 & 276 & 687 & 147 & 1,116 & 849 \\
\hline & Facet joint injection & 17 & 28 & 2,641 & 61 & 4 & 118 & 84 \\
\hline & Hickman line insertion & 18 & 34 & 696 & 75 & 4 & 510 & 87 \\
\hline & Nephrostomy & 20 & 25 & 332 & 399 & 57 & 1,630 & 404 \\
\hline & Oesophageal stent & 12 & 21 & 165 & 249 & 51 & 696 & 298 \\
\hline & Pacemaker (permanent) & 24 & 63 & 4,475 & 307 & 103 & 670 & 358 \\
\hline & PTCA 1 stent & 10 & 35 & 5,444 & 516 & 84 & 882 & 675 \\
\hline
\end{tabular}

DRL, diagnostic reference level; PTCA, percutaneous transluminal coronary angioplasty.

Table 2. Diagnostic reference levels for interventional procedures in the US (2012)

\begin{tabular}{|c|c|c|c|c|}
\hline Procedure & Number & Dose (Gy) & $\mathrm{DAP}\left(\mathrm{Gy} \cdot \mathrm{cm}^{2}\right)$ & Fluoroscopy time (s) \\
\hline Transjugular intrahepatic portosystemic shunt creation & 300 & 3 & 525 & 3,600 \\
\hline Biliary drainage & 20 & 1.4 & 100 & 1,800 \\
\hline Nephrostomy for obstruction & 12 & 0.4 & 40 & 900 \\
\hline Nephrostomy for stone access & 14 & 0.7 & 60 & 1,500 \\
\hline Pulmonary angiography & 215 & 0.5 & 110 & 600 \\
\hline Inferior vena cava filter placement & 40 & 0.25 & 60 & 240 \\
\hline Renal or visceral angioplasty without stent & 210 & 2 & 200 & 1,200 \\
\hline Renal or visceral angioplasty with stent & 200 & 2.3 & 250 & 1,800 \\
\hline Iliac angioplasty without stent & 300 & 1.25 & 250 & 1,200 \\
\hline Iliac angioplasty with stent & 350 & 1.9 & 300 & 1,500 \\
\hline Bronchial artery embolization & 450 & 2 & 240 & 3,000 \\
\hline Hepatic chemoembolization & 300 & 1.9 & 400 & 1,500 \\
\hline Uterine fibroid embolization & 450 & 3.6 & 450 & 2,160 \\
\hline Other tumor embolization & 325 & 2.6 & 390 & 2,100 \\
\hline Gastrointestinal hemorrhage localization and treatment & 425 & 3.8 & 520 & 2,100 \\
\hline Embolization in the head for AVM & 1,500 & 6 & 550 & 8,100 \\
\hline Embolization in the head for aneurysm & 1,350 & 4.75 & 360 & 5,400 \\
\hline Embolization in the head for tumor & 1,700 & 6.2 & 550 & 12,000 \\
\hline Vertebroplasty & 120 & 2 & 120 & 1,260 \\
\hline Pelvic artery embolization for trauma or tumor & 550 & 2.5 & 550 & 2,100 \\
\hline Embolization in the spine for AVM or tumor & 1,500 & 8 & 950 & 7,800 \\
\hline
\end{tabular}

DAP, dose area products; AVM, arteriovenous malformation. 
of approximately 2,100 procedures of the medical institutions participating in the study. The radiation dose data were examined for the DAP, RAK, fluoroscopy time, and number of images. The age, height, and weight of a patient were recorded for each procedure performed, and test cases for each procedure were selected to reduce the deviation caused by the size of the patient. Thus, approximately, 1,960 out of the 2,100 cases of procedures were used for the statistical analysis of the data. Table 2 lists the third quartile DRL values of the DAP and fluoroscopy time for each interventional procedure analyzed in the study in the US. These third quartile values are determined as the national patient DRLs for US interventional procedures. ${ }^{22)}$

\section{Spain}

DRL studies in Spain are conducted by the National Society of Interventional Radiology (NSIR) and the Spanish Nuclear Safety Council (SNSC). In Spain, patient dose surveys and DRL determination studies for interventional radiology are conducted under the European Medical Exposures Directive, which recommends DRL determination. ${ }^{23)}$

In a 2008 study by the NSIR, a survey was conducted with interventional radiologists and medical physicists of ten public hospitals nationwide. ${ }^{24)}$ The survey was performed by seven procedures with the highest frequency, including the biliary drainage and hepatic chemoembolization. Thus, approximately 1,400 cases of the procedures were investigated. Information was collected on the DAP, fluoroscopy time, patient gender, age, size, and weight. In addition, the information on the level of Complexity of the procedures (CI) to determine its difficulty was also classified into three levels (low, medium, and high). However, the study determined that the number of samples collected was not sufficient, and thus, the complexity level was not used. In the investigation, the overall DAP distribution of interventional procedures was $0.6-1136 \mathrm{~Gy} \cdot \mathrm{cm}^{2}$. The fluoroscopy time distribution ranged from 0.3 to 80 minutes. The number of digital subtraction angiography (DSA) image distributions ranged from 0 to 893 images. The DRL was set as the third quartile of the DAP, fluoroscopy time, and number of DSA images. Table 3 summarizes the results of determining the interventional procedure DRL in Spain in 2008.

In 2016, the SNSC conducted a study to reestablish the DRL. ${ }^{25)}$ In this study, senior interventionists and medical physicists at eight university hospitals were surveyed. Seven procedures, including biliary drainage and hepatic chemoembolization, were performed, and approximately 1,600 cases were surveyed over three years from 2010 to 2013. The DRL was reset to the third quartile of the surveyed DAP, fluoroscopy time, and DSA image number distribution. Table 4 lists the results of determining the interventional radiology DRL in Spain in 2016.

Table 3. Dose area products (DAPs) and fluoroscopy times for interventional procedures in Spain (2008)

\begin{tabular}{|c|c|c|c|c|c|c|}
\hline Dose Index & Procedure & Number & Maximum & Minimum & Mean & DRL \\
\hline \multirow[t]{7}{*}{ DAP $\left(\mathrm{Gy} \cdot \mathrm{cm}^{2}\right)$} & Fistulography & 180 & 83 & 0.6 & 9 & 12 \\
\hline & Lower limb arteriography & 685 & 608 & 2.4 & 58 & 73 \\
\hline & Renal arteriography & 55 & 397 & 7.7 & 75 & 89 \\
\hline & Biliary drainage & 205 & 428 & 0.6 & 61 & 80 \\
\hline & Hepatic chemoembolization & 151 & 830 & 27.4 & 216 & 289 \\
\hline & Iliac stent & 70 & 1,136 & 14.1 & 91 & 94 \\
\hline & Uterine embolization & 45 & 677 & 26.6 & 170 & 236 \\
\hline \multirow{7}{*}{$\begin{array}{l}\text { Fluoroscopy } \\
\text { time (s) }\end{array}$} & Fistulography & 180 & 3,120 & 18 & 150 & 150 \\
\hline & Lower limb arteriography & 685 & 3,408 & 30 & 180 & 198 \\
\hline & Renal arteriography & 55 & 1,650 & 18 & 336 & 516 \\
\hline & Biliary drainage & 205 & 4,764 & 36 & 870 & 1,188 \\
\hline & Hepatic chemoembolization & 151 & 4,800 & 168 & 1,188 & 1,458 \\
\hline & Iliac stent & 70 & 4,026 & 78 & 552 & 654 \\
\hline & Uterine embolization & 45 & 2,670 & 18 & 1,428 & 1,818 \\
\hline
\end{tabular}

DRL, diagnostic reference level. 
Table 4. Dose area products (DAPs) and fluoroscopy times for interventional procedures in Spain (2016)

\begin{tabular}{lccrr}
\multicolumn{1}{c}{ Procedure } & Number & $\begin{array}{c}\text { Mean DAP } \\
\left(\mathrm{Gy} \cdot \mathrm{cm}^{2}\right)\end{array}$ & $\begin{array}{c}\text { 3th Q DAP } \\
\left(\mathrm{Gy} \cdot \mathrm{cm}^{2}\right)\end{array}$ & $\begin{array}{c}\text { 3th Q fluoroscopy } \\
\text { time }(\mathrm{s})\end{array}$ \\
\hline Lower extremity arteriography & 784 & - & - & - \\
Renal arteriography & 37 & - & 45 & 340 \\
Transjugular hepatic biopsies & 30 & 30.8 & 30.8 & 30 \\
Biliary drainage & 314 & 189.6 & 214 & 1,038 \\
Uterine fibroid embolization & 56 & 120.2 & 169 & 2,790 \\
Colon endoprostheses & 31 & 216.1 & 303 & 1,578 \\
Hepatic chemoembolization & 269 & 83.9 & 119 & 1,800 \\
Femoropopliteal revascularization & 62 & 105.7 & & 1,284 \\
Iliac stent & 66 & & & \\
\hline
\end{tabular}

Table 5. Dose area products (DAPs) for interventional procedures in Germany (2019)

\begin{tabular}{|c|c|c|c|c|c|}
\hline \multirow{2}{*}{ Procedure } & \multicolumn{4}{|c|}{$\mathrm{DAP}\left(\mathrm{Gy} \cdot \mathrm{cm}^{2}\right)$} & \multirow{2}{*}{$\mathrm{D}_{\text {eff }}(\mathrm{mSv})$} \\
\hline & 25 th & 50th & 75th & DRL & \\
\hline $\begin{array}{l}\text { Thrombus aspiration after stroke } \\
\text { (recanalization of cerebral arteries) }\end{array}$ & 51 & 91 & 158 & 180 & 11 \\
\hline $\begin{array}{l}\text { Coiling of a cerebral aneurysm } \\
\text { (EVAR of the cerebral artery) }\end{array}$ & 74 & 121 & 192 & 250 & 16 \\
\hline PCI & 20 & 34 & 49 & 48 & 9 \\
\hline Combined CA and PCI & 28 & 40 & 55 & 55 & 10 \\
\hline TAVI & 25 & 49 & 82 & 80 & 15 \\
\hline \multicolumn{6}{|l|}{ EVAR } \\
\hline For thoracic aorta & 47 & 114 & 203 & 230 & 28 \\
\hline For infrarenal abdominal aorta & 55 & 108 & 203 & & 32 \\
\hline For suprarenal abdominal aorta & 47 & 95 & 218 & & 36 \\
\hline TACE & 62 & 121 & 224 & 230 & 392 \\
\hline \multicolumn{6}{|l|}{ PTA } \\
\hline For pelvis & 22 & 44 & 87 & 90 & 23 \\
\hline For thigh and knee & 8 & 15 & 35 & 40 & 10 \\
\hline For lower leg and food & 6 & 10 & 20 & 25 & 6 \\
\hline
\end{tabular}

DRL, diagnostic reference level; $\mathrm{D}_{\text {eff }}$ effective dose; EVAR, endovascular treatment of abdominal aortic aneurysms; PCI, percutaneous coronary intervention; CA, coronary angiography; TAVI, transcatheter aortic valve implantation; TACE, transcatheter arterial chemoembolization; PTA, percutaneous transluminal angioplasty.

\section{Germany}

In Germany, research on medical exposure status and DRL determination has been conducted, with focus on the Federal Office for Radiation Protection (BfS). The BfS revised the radiation-related regulations in 2003 to recommend the establishment of DRLs in diagnostic radiographs. Since then, periodic investigations and DRL determination of various medical radiation have been performed. The DRL for interventional radiology was set based on numerous databases collected nationally from the "ärztliche Stellen" (the Quality Assurance Commission), Ger- man Institute of Interventional Radiology (DeGIR), and AQUA Institute. In Germany, the DRLs were determined for interventional radiology in 2003, 2016, and 2019 based on the collected data. ${ }^{26-28)}$ In 2003, two procedures of the percutaneous transluminal angioplasty (PTA) and percutaneous transluminal coronary angioplasty were set. In 2016 and 2019, 12 and 11 procedures were announced, including the percutaneous coronary intervention, transcatheter aortic valve implantation, transcatheter arterial chemoembolization, endovascular treatment of abdominal aortic aneurysms, and PTA. Table 5 and 6 summarize Germany's interventional radiology DRL as of 2019. ${ }^{28)}$ 


\section{Switzerland}

Swiss medical radiation survey research is conducted by the Swiss Federal Office of Public Health. In the early 2000s, the Federal Office of Public Health, in collaboration with two university radiation physics laboratories formed a working group for the radiation protection optimization of X-ray examination. The purpose of this group was to generate awareness of the radiation risks to physicians and radiologists to optimize dose-intensive radiographs. In 2007, DRL determination was performed for interventional radiology and diagnostic fluoroscopy ${ }^{29)}$ In this study, approximately 1,000 procedures were investigated in the radiology and cardiac disease departments of nine national and private hospitals for two years. Twelve procedures were investigated in total, and among these, six types of interventional radiology were examined, including hepatic embolization, biliary drainage, and stent insertion. The radiation dose information was examined for the DAP, fluoroscopy time, and number of images. Table 7 presents the DRL for Switzerland in 2007. ${ }^{29)}$

\section{Finland}

Research on medical radiation in Finland is conducted by the Radiation and Nuclear Safety Authority (STUK). The STUK measures or evaluates the patient doses every three years to establish the interventional radiology DRL. Finland has presented a DRL for interventional radiology in 2016. ${ }^{30)}$ In Finland, in interventional radiology and cardiology procedures, the difficulty of the procedure may have a larger impact on the radiation dose than on the procedure steps or procedure equipment. Thus, it is recommended to use the radiation doses of numerous patients to set the DRL and consider the difficulties in the procedure, if necessary. Table 8 presents the DRL for interventional radiology in Finland conducted in 2016.

\section{United Nations Scientific Committee on the Effects of Atomic Radiation}

The United Nations Scientific Committee on the Effects of Atomic Radiation (UNSCEAR) is an international organization established by the United Nations (UN) for the analysis of the effects and risks of radiation on the human body. UNSCEAR collects information on the medical radiation exposure doses in all the countries to understand the current status of medical radiation safety management. The information is gathered by collecting questionnaires from representatives of each country or by researching national

Table 6. Fluoroscopy times for interventional procedures in Germany (2019)

\begin{tabular}{|c|c|c|c|}
\hline \multirow{2}{*}{ Procedure } & \multicolumn{3}{|c|}{ Fluoroscopy time (s) } \\
\hline & 25th & 50th & 75th \\
\hline Thrombus aspiration after stroke (recanalization of cerebral arteries) & 720 & 1,260 & 2,100 \\
\hline Coiling of a cerebral aneurysm (EVAR of the cerebral artery) & 1,260 & 2,040 & 3,240 \\
\hline PCI & 312 & 558 & 780 \\
\hline Combined CA and PCI & 354 & 570 & 780 \\
\hline TAVI & 474 & 720 & 1,080 \\
\hline \multicolumn{4}{|l|}{ EVAR } \\
\hline For thoracic aorta & 420 & 720 & 1,140 \\
\hline For infrarenal abdominal aorta & 840 & 1,260 & 1,980 \\
\hline For suprarenal abdominal aorta & 840 & 1,560 & 3,120 \\
\hline TACE & 660 & 1,020 & 1,500 \\
\hline \multicolumn{4}{|l|}{ PTA } \\
\hline For pelvis & 420 & 600 & 1,020 \\
\hline For thigh and knee & 420 & 660 & 1,080 \\
\hline For lower leg and food & 540 & 1,020 & 1,860 \\
\hline
\end{tabular}

EVAR, endovascular treatment of abdominal aortic aneurysms; PCI, percutaneous coronary intervention; CA, coronary angiography; TAVI, transcatheter aortic valve implantation; TACE, transcatheter arterial chemoembolization; PTA, percutaneous transluminal angioplasty. 
Table 7. Dose area products (DAPs) and fluoroscopy times for interventional procedures in Switzerland (2007)

\begin{tabular}{|c|c|c|c|c|}
\hline Category & Procedure & $\begin{array}{l}\text { Fluoroscopy } \\
\text { time (min) }\end{array}$ & Frame (n) & $\mathrm{DAP}\left(\mathrm{Gy} \cdot \mathrm{cm}^{2}\right)$ \\
\hline \multirow[t]{6}{*}{ Diagnostic } & Barium meal & 7 & 40 & 60 \\
\hline & Lower limb and iliac angiography & 8 & 150 & 210 \\
\hline & Cerebral angiography & 15 & 480 & 1,215 \\
\hline & Barium enema & 10 & 25 & 150 \\
\hline & Coronary angiography & 7 & 1,400 & 80 \\
\hline & Electrophysiology & 1 & 480 & 10 \\
\hline \multirow[t]{6}{*}{ Interventional } & Hepatic embolization & 30 & 160 & 620 \\
\hline & Biliary drainage and stent insertion & 25 & 30 & 240 \\
\hline & Cerebral embolization & 50 & 800 & 440 \\
\hline & Iliac dilatation and stent insertion & 25 & 200 & 460 \\
\hline & Percutaneous coronary intervention & 20 & 1,500 & 110 \\
\hline & Cardiac thermo-ablation & 20 & 800 & 140 \\
\hline \multirow[t]{2}{*}{ Combined } & $\begin{array}{l}\text { Coronary angiography+ } \\
\text { percutaneous coronary intervention }\end{array}$ & 20 & 2,800 & 260 \\
\hline & Electrophysiology+cardiac thermo-ablation & 30 & 0 & 280 \\
\hline
\end{tabular}

Table 8. Diagnostic reference levels (DRLs) for interventional procedures in Finland (2016)

\begin{tabular}{lcr}
\hline \multicolumn{1}{c}{ Procedure } & DAP $\left(\mathrm{Gy} \cdot \mathrm{cm}^{2}\right)$ & Fluoroscopy time $(\mathrm{min})$ \\
\hline Coronary artery examination using a contrast medium & 30 & 4 \\
Percutaneous coronary intervention* & 75 & 15 \\
Pacemaker installation (excluding CRT pacemaker installation) & 3.5 & 5 \\
Transcatheter aortic valve implantation & 90 & 19 \\
Electrophysiological treatment of atrial fibrillation & 25 & 12 \\
CRT pacemaker installation & 22 & - \\
Electrophysiological treatment of atrial flutter & 16 & - \\
Electrophysiological treatment of atrioventricular nodal reentrant tachycardia & 6 & - \\
\hline
\end{tabular}

DAP, dose area products; CRT, cardiac resynchronization therapy.

*Including potential angiography (ad hoc percutaneous coronary intervention).

research reports and literatures. The UNSCEAR recently published a report on the results of worldwide medical radiation exposure surveys obtained from countries and institutions during 1997-2007. ${ }^{1)}$ In this study, although the interventional radiology DRL was not set, the distributions and differences in the DAPs for different countries at a particular procedure were analyzed by the UNSCEAR. The UNSCEAR collected the results of the DAP and effective dose evaluation for 30 procedures regarding interventional radiology according to five major categories of biopsy, biliary tract, and urinary system. In the 2010 report, the DAP collected from each country was presented in each literature. Table 9 lists the distribution of the DAP according to the interventional radiology reported by the UNSCEAR.

\section{Korea}

Survey research on the actual conditions of patient dose in the interventional radiation procedures in Korea is actively conducted with the MFDS. The MFDS set a DRL based on the collected information of the radiation dose of the interventional radiology performed in 2007 and 2012. In the 2012 study, 11 procedures were selected from among the interventional radiology procedures performed in Korea, which were relatively numerous procedures or were of high importance in relation to the radiation protection, including the TACE, arteriovenous fistula (AVF), percutaneous transluminal angioplasty with stent in arteries of the lower extremity (LE PTA and stent). The study collected patient radiation dose data from the eleven interventional 
Table 9. Dose distributions for interventional procedures in the United Nations Scientific Committee on the Effects of Atomic Radiation (2010)

\begin{tabular}{|c|c|c|c|}
\hline Category & Procedure & $\mathrm{DAP}\left(\mathrm{Gy} \cdot \mathrm{cm}^{2}\right)$ & Effective dose (mSv) \\
\hline \multirow[t]{4}{*}{ Biopsy } & Pathological specimen & - & 1.6 \\
\hline & Biopsy & 6 & 1.6 \\
\hline & Small bowel biopsy & 1 & 0.26 \\
\hline & Venous sampling & - & 0.4 \\
\hline \multirow[t]{8}{*}{ Biliary and urinary systems } & Bile duct drainage & $38-150$ & $9.9-38$ \\
\hline & Bile duct dilation/Stenting & $43-54$ & $11-14$ \\
\hline & Biliary intervention & 54 & 14 \\
\hline & Biliary duct stone extraction & 27 & 7 \\
\hline & Lithotripsy & 5 & 1.3 \\
\hline & Nephtostomy & $8-56$ & $2.1-15$ \\
\hline & Ureteric stenting & 18 & 4.7 \\
\hline & Kidney stent insertion & 49 & 12.7 \\
\hline \multirow[t]{14}{*}{ Cardiovascular } & Emboliztion & $75-114$ & $20-30$ \\
\hline & Management of caricocele & $50.8-131$ & $6.4-38$ \\
\hline & Neuroembolization & $81-320$ & $2.3-11$ \\
\hline & Thrombolysis & 13.5 & 3.5 \\
\hline & TIPS & $77-524$ & $20-87$ \\
\hline & Valvuloplasty & 162 & 29 \\
\hline & Vascular stenting & $40-42$ & $5.8-10$ \\
\hline & Pelvic vein embolization & - & 60 \\
\hline & Insertion of caval filters & 48 & 13 \\
\hline & Removal of foreings bodies & - & 7 \\
\hline & Coronary angiography & $12.7-147$ & $3.1-16$ \\
\hline & PTCA & $11.8-205$ & $5.4-41$ \\
\hline & Stent procedures & $41-166$ & $7-13$ \\
\hline & Pacemaker insertion & $8.46-19$ & - \\
\hline Uterine fibroid embolization & Uterine fibroid embolization & $30.6-298$ & $8-78$ \\
\hline \multirow[t]{6}{*}{ Gastroinstinal } & Feeding tube & 13 & 3.4 \\
\hline & Gastrostomy & 13 & 3.4 \\
\hline & Diliation/stenting oesophagus & 15 & 1.5 \\
\hline & Dilation pyloric stenosis & 27 & 7 \\
\hline & Colonic stent & - & 7 \\
\hline & Nerve injection & 1.7 & 0.2 \\
\hline
\end{tabular}

DAP, dose area products; TIPS, transjugular intrahepatic portosystemic shunt; PTCA, percutaneous transluminal coronary angioplasty.

radiology procedures selected from 24 major hospitals nationwide. The data surveys were performed according to the DAP and fluoroscopy time. In addition, 8,415 domestic data were obtained for each procedure, and the DRL was presented as the third quartile of the survey results. ${ }^{10)}$ In addition, the accuracy of the built-in DAP meter of the IR equipment installed in Korea was evaluated, and the actual effects of the dose reduction parameters were evaluated by phantom measurement experiments.

Table 10 lists the results of the 2012 Korean interventional radiology DRL study. ${ }^{10)}$ The DAP distribution for each procedure is found to be $0.01-3081 \mathrm{~Gy} \cdot \mathrm{cm}^{2}$ overall, with 8.5-1679 $\mathrm{Gy} \cdot \mathrm{cm}^{2}$ for the TACE and $0.1-686 \mathrm{~Gy} \cdot \mathrm{cm}^{2}$ for the transfemoral cerebral angiography (TFCA) procedure reprehensively. The fluoroscopy time distribution is overall $2-16,878$ seconds, with $32-5,775$ seconds for the TACE and 16-6,636 seconds for the TFCA procedure reprehensively. Even for the same procedure, the difference is more than several hundred times for the performer, patient, and hospital. The DRL is set to the third quartile of the DAP and fluoroscopy time distributions for each procedure. Representatively, the DRLs for the TACE and TFCA are 238 and 
Table 10. Dose area products (DAPs) and fluoroscopy times for interventional procedures in Korea (2012)

\begin{tabular}{|c|c|c|c|c|c|c|c|c|}
\hline \multirow{2}{*}{$\begin{array}{l}\text { Radiation } \\
\text { dose units }\end{array}$} & \multirow{2}{*}{ Procedure } & \multicolumn{3}{|c|}{ Measurement (n) } & \multirow{2}{*}{ Mean } & \multirow{2}{*}{ Minimum } & \multirow{2}{*}{ Maximum } & \multirow{2}{*}{ DRL } \\
\hline & & Hospital & Room & Patient & & & & \\
\hline \multirow[t]{11}{*}{$\mathrm{DAP}\left(\mathrm{Gy} \cdot \mathrm{cm}^{2}\right)$} & TACE & 22 & 35 & 3,139 & 191.1 & 8.5 & $1,678.8$ & 237.7 \\
\hline & AVF & 9 & 14 & 234 & 14.1 & 0.2 & 113.1 & 17.3 \\
\hline & LE PTA \& Stent & 13 & 16 & 278 & 85.4 & 1.3 & 571.0 & 114.1 \\
\hline & TFCA & 18 & 29 & 2,083 & 149.6 & 0.1 & 685.5 & 188.5 \\
\hline & Aneurysm coil & 15 & 18 & 320 & 316.9 & 35.8 & $3,080.5$ & 383.5 \\
\hline & PTBD & 15 & 25 & 566 & 36.1 & 0.3 & $1,290.8$ & 37.5 \\
\hline & Biliary Stent & 21 & 36 & 118 & 47.4 & 0.7 & 289.4 & 64.6 \\
\hline & PCN & 13 & 15 & 219 & 19 & 0.5 & 189.1 & 22.4 \\
\hline & Hickman & 15 & 20 & 519 & 4 & 0.03 & 75.3 & 4.3 \\
\hline & Chemoport & 20 & 24 & 479 & 2.1 & 0.01 & 31.7 & 2.8 \\
\hline & Perm-cath & 17 & 17 & 460 & 3.7 & 0.02 & 37.4 & 4.4 \\
\hline \multirow{11}{*}{$\begin{array}{l}\text { Fluoroscopy } \\
\text { time (s) }\end{array}$} & TACE & 22 & 35 & 3,139 & 935.6 & 32.0 & $5,775.0$ & $1,224.0$ \\
\hline & AVF & 9 & 14 & 234 & 649.8 & 30.0 & $3,715.0$ & 786.1 \\
\hline & LE PTA \& Stent & 13 & 16 & 278 & $1,090.8$ & 42.0 & $5,897.0$ & $1,460.4$ \\
\hline & TFCA & 18 & 29 & 2,083 & 599.9 & 16.0 & $6,636.0$ & 686.0 \\
\hline & Aneurysm Coil & 15 & 18 & 320 & $2,325.5$ & 250.0 & $16,878.0$ & $2,975.8$ \\
\hline & PTBD & 15 & 25 & 566 & 379.3 & 16.0 & $6,777.0$ & 427.5 \\
\hline & Biliary Stent & 21 & 36 & 118 & 606.5 & 30.0 & $2,493.0$ & 759.8 \\
\hline & PCN & 13 & 15 & 219 & 222.9 & 8.0 & $1,643.0$ & 236.0 \\
\hline & Hickman & 15 & 20 & 519 & 51.3 & 5.0 & 987.0 & 54.0 \\
\hline & Chemoport & 20 & 24 & 479 & 34.1 & 5.0 & 428.4 & 43.0 \\
\hline & Perm-cath & 17 & 17 & 460 & 56.3 & 2.0 & 507.0 & 67.8 \\
\hline
\end{tabular}

DRL, diagnostic reference level; TACE, transcatheter arterial chemoembolization; AVF, arteriovenous fistula; TFCA, transfemoral cerebral angiography; PTBD, percutaneous transhepatic biliary drainage; PCN, percutaneous nephrostomy.
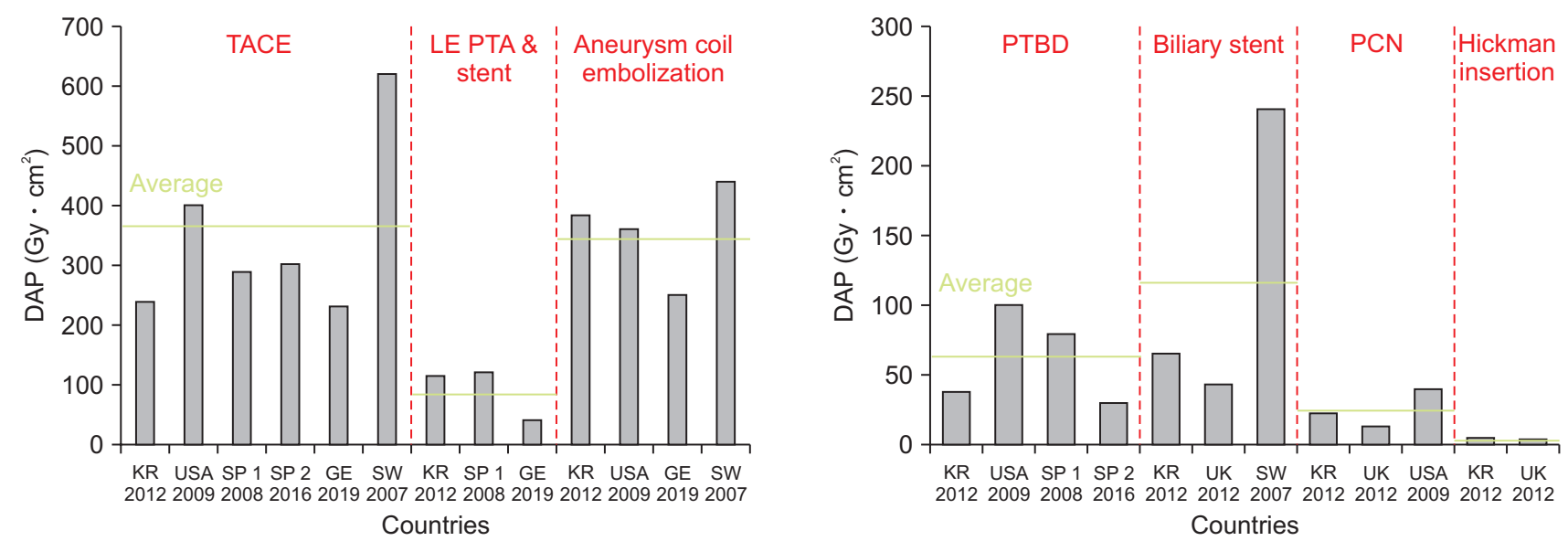

Fig. 5. Dose area products (DAPs) for interventional procedures by country. TACE, transcatheter arterial chemoembolization; PTBD, percutaneous transhepatic biliary drainage; PCN, percutaneous nephrostomy.

$189 \mathrm{~Gy} \cdot \mathrm{cm}^{2}$ for the DAP and 1,224 and 686 seconds for the fluoroscopy time, respectively.

Fig. 5 and 6 display the comparison of the DAPs and fluoroscopy times in domestic and international inter- ventional procedures. The domestic DRL is generally low compared to the DRLs in other countries. Comparing the DRL for the domestic DAP with the average of that in other countries, the domestic DRL is found to be about two times 

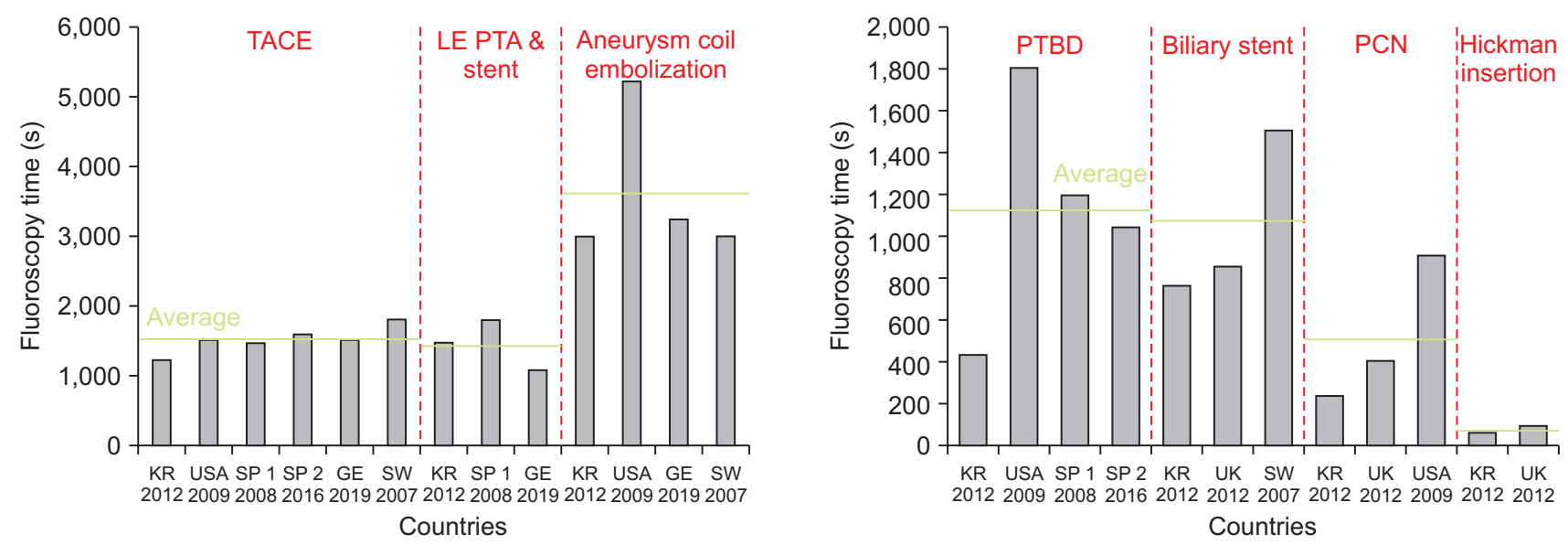

Fig. 6. Fluoroscopy times for interventional procedures by country. TACE, transcatheter arterial chemoembolization; PTBD, percutaneous transhepatic biliary drainage; PCN, percutaneous nephrostomy.

lower for the percutaneous transhepatic biliary drainage (PTBD) and biliary stent procedures. Further, for the TACE and percutaneous nephrostomy (PCN) procedures, it is approximately $55 \%$ and $18 \%$ lower, respectively. For the LE PTA and stent, aneurysm coil embolization, and Hickman insertion procedures, it is approximately $30 \%, 8 \%$, and $30 \%$ higher, respectively. Further, when comparing the DRL for the fluoroscopy time with the average of the other countries, the domestic DRL is lower, except for the LE PTA and stent procedure, which is at a similar level. It is about approximately thrice lower for the PTBD and PCN procedures and approximately 50\% lower for the biliary stent and Hickman insertion procedures. Moreover, it is approximately $30 \%$ lower for the TACE and aneurysm coil embolization procedures, and is similar to that of the other countries for the LE PTA and stent procedures.

\section{Conclusions}

The use of interventional radiology is rapidly increasing owing to the increase in its demand arising from the average lifespan increase and technology development. It is known that interventional radiology takes longer than other radiographies and uses relatively high radiation doses. Considering the increased usage and high radiation dose of these interventional procedures, the radiation protection of a patient and patient dose management are important during interventional procedures. Under these circumstances, we investigated the current status of the
DRL setting in domestic and foreign interventional radiology procedures. Further, they were compared and analyzed to understand the radiation dose exposure status and patient dose management in interventional radiology for the Korean population.

In Korea, a study on setting the interventional radiology DRL was conducted in 2012 by the Ministry of Food and Drug Safety. In this study, DRLs were set for the DAPs and fluoroscopy times of eleven procedures, which relatively have numerous procedures or are of high importance in relation to radiation protection. They included the TACE, AVF, and LE PTA and stent, among interventional radiology performed in Korea. Representatively, the DRLs for the TACE and TFCA were 237.7 and $188.5 \mathrm{~Gy} \cdot \mathrm{cm}^{2}$, respectively, and for the fluoroscopy time, 1,224 and 686 seconds, respectively. When comparing the domestic interventional radiology DRL with those of other countries, the domestic DRL was found to be low, in general. When comparing the DRL for the domestic DAP with the average values of the other countries, for the PTBD, biliary stent, TACE, and PCN procedures, it was found to be as low as $20 \%$ to as high as nearly twice the average values of the other countries. Further, for the LE PTA and stent, aneurysm coil embolization, and Hickman insertion procedures, it was approximately $30 \%, 8 \%$, and $30 \%$ higher, respectively. In addition, when comparing the DRL for the fluoroscopy time with the average of the other countries, the domestic DRL was lower, except for the LE PTA and stent procedures, which were at similar levels. 
Interventional radiology, as medical radiation exposure, benefits a patient and is an important procedure that is directly related to the life of a patient; thus, it is not appropriate to perform extremely strict management of the radiation exposure that a patient receives without considering the overall circumstances. However, to optimize the procedure and reduce patient dose, it is necessary to understand the current status of domestic patient dose management by investigating the patient exposure level and DRL setting during the interventional procedure. In the future, if the nationwide survey is continuously conducted for medical radiation safety management and patient protection, it will ultimately contribute to maximizing the benefit to patients from interventional radiology while minimizing the radiation dose of the patient.

\section{Acknowledgements}

This work was supported by the Nuclear Safety Research Program through the Korea Foundation of Nuclear Safety (KoFONS) using the financial resource granted by the Nuclear Safety and Security Commission (NSSC) of the Republic of Korea (No. 1803013).

\section{Conflicts of Interest}

The authors have nothing to disclose.

\section{Availability of Data and Materials}

All relevant data are within the paper and its Supporting Information files.

\section{References}

1. UNSCEAR 2008 Report. Sources and effects of ionizing radiation. UNSCEAR Report. Vienna: UNSCEAR. 2010; 2008.

2. Study on the radiation exposure of the workers of the interventional radiology. Seoul: Ministry of Food and Drug Safety; 2013.

3. ICRP Publication 60: 1990 Recommendations of the International Commission on Radiological Protection. Ottawa: International Commission on Radiological Protection;
1991.

4. ICRP Publication 73: Radiological protection and safety in medicine. Ottawa: International Commission on Radiological Protection; 1991.

5. ICRP Publication 105: Radiological protection in medicine. Ottawa: International Commission on Radiological Protection; 2007.

6. ICRP Publication 135: Diagnostic reference levels in medical imaging. Ottawa: International Commission on Radiological Protection; 2017.

7. ICRP Supporting Guidance 2: Radiation and your patient: a guide for medical practitioners. Ottawa: International Commission on Radiological Protection; 2002.

8. IAEA Safety Series No. 115: International basic safety standards for protection against ionizing radiation and for the safety of radiation sources. Vienna: International Commission on Radiological Protection; 1996.

9. Evaluation of patient dose in interventional radiology. Seoul: Ministry of Food and Drug Safety; 2007.

10. Evaluation of patient doses from interventional radiology procedures. Seoul: Ministry of Food and Drug Safety; 2012.

11. HPA-CRCE-034: Doses to patients from radiographic and fluoroscopic x-ray imaging procedures in the UK - 2010 review. London: Health Protection Agency; 2012.

12. ICRU Report 74: Patient dosimetry for $X$ rays used in medical imaging. Bethesda: International Commission in Radiation Units and Measurement; 2005.

13. IAEA Technical Report Series No. 457: Dosimetry in diagnostic radiology: an international code of practice. Vienna: International Atomic Energy Agency; 2007.

14. IEC 60532: Radiation protection instrumentation - installed dose rate meters, warning assemblies and monitors - $\mathrm{X}$ and gamma radiation of energy between $50 \mathrm{keV}$ and 7 MeV. Geneva: International Electrotechnical Commission; 2010.

15. ICRP Publication 120: Radiological protection in cardiology. Ottawa: International Commission on Radiological Protection; 2013.

16. Fletcher DW, Miller DL, Balter S, Taylor MA. Comparison of four techniques to estimate radiation dose to skin during angiographic and interventional radiology procedures. J Vasc Interv Radiol. 2002;13:391-397. 
17. Chida K, Saito H, Otani H, Kohzuki M, Takahashi S, Yamada S, et al. Relationship between fluoroscopic time, dose-area product, body weight, and maximum radiation skin dose in cardiac interventional procedures. AJR Am J Roentgenol. 2006;186:774-778.

18. NCRP Report No. 168: Radiation dose management for fluoroscopically-guided interventional medical procedures. Bethesda: National Council on Radiation Protection \& Measurements; 2010.

19. Chambers CE, Fetterly KA, Holzer R, Lin PJ, Blankenship JC, Balter S, et al. Radiation safety program for the cardiac catheterization laboratory. Catheter Cardiovasc Interv. 2011;77:546-556.

20. HPA-RPD-029: Doses to patients from radiographic and fluoroscopic X-ray imaging procedures in the UK-2005 review. London: Health Protection Agency; 2007.

21. Miller DL, Kwon D, Bonavia GH. Reference levels for patient radiation doses in interventional radiology: proposed initial values for U.S. practice. Radiology. 2009;253:753764.

22. NCRP Report 172: Reference levels and achievable doses in medical and dental imaging : recommendations for the United States. Bethesda: National Council on Radiation Protection \& Measurements; 2012.

23. 97/43/ EURATOM: The European directive on health protection of individuals against the dangers of ionising radiation in relation to medical exposures. Rome: EURopean ATOMic Energy Community; 1997.
24. Vano E, Sanchez R, Fernandez JM, Gallego JJ, Verdu JF, de Garay MG, et al. Patient dose reference levels for interventional radiology: a national approach. Cardiovasc Intervent Radiol. 2009;32:19-24.

25. Ruiz-Cruces R, Vano E, Carrera-Magariño F, MorenoRodriguez F, Soler-Cantos MM, Canis-Lopez M, et al. Diagnostic reference levels and complexity indices in interventional radiology: a national programme. Eur Radiol. 2016;26:4268-4276.

26. Bundesamt für Strahlenschutz: Bekanntmachung der aktualisierten diagnostischen Referenzwerte für nuklearmedizinische Untersuchunge. Salzgitter: Bundesamt für Strahlenschutz; 2003.

27. Bekanntmachung der aktualisierten diagnostischen Referenzwerte für diagnostische und interventionelle Röntgenuntersuchungen. Salzgitter: Bundesamt für Strahlenschutz; 2016.

28. Schegerer AA, Loose R, Heuser L, Brix G. Diagnostic reference levels for diagnostic and interventional X-ray procedures in Germany: update and handling. Rofo. 2019;191: 739-751.

29. Aroua A, Rickli H, Stauffer JC, Schnyder P, Trueb PR, Valley JF, et al. How to set up and apply reference levels in fluoroscopy at a national level. Eur Radiol. 2007;17:1621-1633.

30. Reference levels for the patient's radiation exposure in cardiology. Helsinki: Radiation and Nuclear Safety Authority; 2016. 